\title{
Sistemas complejos y pensamiento sistémico explorados desde la práctica del arte generativo como marco de aproximación a la complejidad contemporánea
}

\section{Anna Carreras}

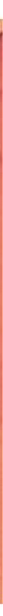

Recibido: 10.05 .2021

Revisado: 14.06 .2021

Publicado: 30.12.2021

Como citar este artículo

Carreras,A., 2021. Sistemas complejos y pensamiento sistémico explorados desde la práctica del arte generativo como marco de aproximación a la complejidad contemporánea. Inmaterial. Diseño, Arte y Sociedad, 6 (12), pp.155-180

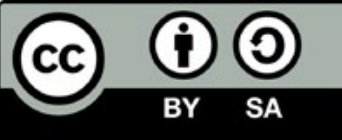




\section{Resumen}

El universo es complejo. Los objetos, sujetos, natura, cultura, colectivos, individuos, sean de la especie que sean; sus decisiones, acciones, evoluciones; presentan complejidades. El arte generativo, digital y basado en instrucciones, aunque parezca contradictorio debido a su naturaleza algorítmica, se relaciona con el pensamiento sistémico y con la visión compleja de las cosas. El pensamiento sistémico propone un marco para romper con esquemas modernos de conocimiento y nos acerca a la complejidad de la mano de ideas contemporáneas: ontologías relacionales, pensamiento situado, visiones holísticas, dinámicas y rizomáticas. La práctica artística del arte generativo explora la complejidad de la mano de estas ideas y desde una perspectiva y posición únicas.

Palabras claves: complejidad, sistemas complejos, pensamiento sistémico, sistemas generativos, arte generativo

\section{Abstract}

Our Universe is complex. Objects, subjects, nature, culture, collectives, individuals, from all the species; their decisions, actions, evolutions; each of these things presents complexities. Generative art, although it can initially seem contradictory due to its algorithmic nature, it opens a path to the understanding of complex things. This kind of instruction-based art, it's practice, is related to system thinking which offers a framework to break with modern knowledge schemes. System thinking brings us closer to the idea of complexity merging systems characteristics with contemporary ideas such as relational ontologies, situated thinking and an holistic, dynamic and rhizomatic vision of our Universe.

Keywords: complexity, complex systems, systems thinking, generative systems, generative art 


\section{1.- Introducción}

El universo es un embrollo, una maraña caótica enredada. Es complejo, es dinámico y se encuentra en un estado de transitoriedad hacia otro estado que no estará nunca en equilibrio perfecto. Se organiza y evoluciona, crea diversidad y no uniformidad sin llegar a ser azar puro. "El universo es bello; y la belleza es la opción menos probable de la evolución" (Wagensberg, 1998). El comportamiento de cualquier sistema complejo en la naturaleza, en los ordenadores, en organizaciones sociales, etc. muestra esta complejidad y cuan incompletos son nuestros modelos mentales.

El pensamiento sistémico -el system thinking ${ }^{1-}$, propone mirar y entender el universo y cualquiera de sus partes como un sistema, o subsistema de sistemas. Enfatiza el holismo frente al reduccionismo, destaca el comportamiento de los organismos y elementos del sistema frente al mecanismo y resalta el proceso frente al resultado. En contraste con las aproximaciones epistémicas tradicionales de occidente, el paradigma del pensamiento sistémico no centra su atención en las cualidades absolutas de varias partes sino en la organización del todo en términos de relación, como un proceso dinámico e interactivo entre los elementos que constituyen este todo. Recoge, en cierto modo, la idea de Simon (1981, p184) de que "en los sistemas complejos el conjunto es más que la suma de las partes".

En este artículo se muestra cómo el pensamiento sistémico, heredero de las ideas de la cibernética, propone un marco para aproximarse a la complejidad contemporánea que ofrece al arte, a la arquitectura o al diseño nuevas posibilidades de invención formal y funcional.

El siguiente apartado, número 2, presenta el estado del arte y situa el pensamiento sistémico en su contexto histórico, la cibernética. A continuación, se aborda el concepto de complejidad y se profundiza en los sistemas complejos y sus características. En el apartado 4 se relacionan el pensamiento sistémico, la complejidad y varias propuestas del pensamiento contemporáneo. Posteriormente, en el apartado 5 y 6 se ahonda en las características y la práctica del arte generativo, poniendo

\footnotetext{
${ }^{1}$ Aclaración propia de Shanken (2015. p19) quien puntualiza el uso del concepto pensamiento sistémico en vez de los términos científicos cibernética o teoría de sistemas para referirse a un marco epistémico más genérico.
} 
esta en relación a los sistemas complejos. Finalmente, en el apartado 7 se describe un proyecto extenso de arte generativo, con ejemplos concretos, que muestra como esta investigación basada en la práctica crea un espacio único y coherente en el que la experimentación, el contexto, la reflexión y la creación tienen lugar simultáneamente. El arte generativo propone una aproximación y visión única a camino entre el arte, la tecnología, el pensamiento y la ciencia. Una práctica y una mirada única para explorar la complejidad de la realidad contemporánea.

\section{2.- Cibernética}

La teoría de la información y la teoría de sistemas nutren a la cibernética de mediados del s. XX. La cibernética no se limita a las ciencias o a la ingeniería, en la década de los 60 y 70 cuando se consolida penetra también en el mundo del arte y de la cultura. La cibernética establece una ciencia de la comunicación y el control que parece aplicar tanto a máquinas como a animales y proporciona un modelo teórico para construir sistemas mecánicos que exhiben comportamientos similares a los seres vivos. Proporciona un paradigma para entender ciertos problemas y modelar posibles futuros. En 1968 la exposición "Cybernetic Serendipity”, en el "Institute of Contemporary Arts” de Londres, ya presenta trabajos gráficos trazados con plóter, entornos de luz y sonido con detección y robots. Piezas que anticiparon muchas de las características importantes del medio digital actual. Algunas obras se centraron en la estética de máquinas y transformaciones, otras propuestas eran dinámicas y orientadas a los procesos, explorando las posibilidades de la interacción y de sistemas abiertos como objeto artístico (Paul, 2015).

La primera ola cibernética, surgida hacia 1942, se centró en cómo los sistemas pueden mantener un estado de equilibrio, denominado homeostasis, a partir de caminos de retroalimentación -feedback loopsque permite la auto regulación del sistema. Sistemas de retroalimentación parecidos al termostato que responde a las fluctuaciones de la temperatura encendiendo o apagando el sistema de calefacción o de aire frío. Encontramos estas ideas en trabajos artísticos y ensayos de John Cage, Iannis Xenakis, Brian Eno, Ken Rinaldo, Norman White o Christa Sommerer y Laurent Migneau entre muchos otros, recogidos por Shanken (2015). En campos como la arquitectura, encontramos ideas de la cibernética en trabajos de Pask, que proponen y especulan sobre meta-estructuras urbanas capaces de evolucionar, a través de sis- 
temas de sensores y retroalimentación, conjuntamente con sus habitantes, para mantener el sistema siempre en equilibrio (Shanken, 2015).

En una segunda ola, aparecida a inicios de los años 70, la cibernética se centra en incluir al observador como parte intrínseca del sistema. Los observadores pasan a ser reconocidos como parte fundamental de sus propios experimentos e inseparables de ellos. Esta segunda ola, alimentada por los avances e ideas en biología de Varela y Maturana abraza también el concepto de "autopoiesis", la idea de que todo sistema se organiza respecto a su entorno, su medio, para cambiar y sobrevivir.

A partir de los años 90, numerosos factores incluyendo los avances en ciencia computacional, biología, redes, la aparición de los autómatas celulares y la visualización de datos, facilitan la simulación de ciertos sistemas complejos, llevando a los investigadores a retomar ideas de la cibernética y la teoría de sistemas, lo que podríamos considerar una tercera ola cibernética.

El pensamiento moderno puede asumir que el análisis de estos sistemas cibernéticos, las interconexiones, los datos y la potencia de la computación y los ordenadores puede ser la clave para la predicción, el equilibrio, el control e incluso cierta inteligencia artificial. Sin embargo, la mayoría de sistemas, que presentan cierta complejidad son dinámicos y variantes, no lineales, auto-organizados y con retroalimentación -feedback-; son también inherentemente impredecibles y no se pueden controlar. Se pueden observar y entender de forma genérica, se puede aprender de ellos, pero anticipar su funcionamiento y su comportamiento futuro es imposible. Desde la teoría cuántica a las matemáticas del caos, las recientes teorías apuntan siempre a un universo con incertezas, impredecible y lleno de complejidades. La idea de conseguir que un sistema complejo haga lo que uno desea es una quimera.

Desde el arte y el diseño se pueden proponer proyectos que muestran características complejas, que escapan a los límites de inteligibilidad que proporcionó la modernidad. El arte y el diseño pueden incorporar ideas del pensamiento sistémico junto con ideas contemporáneas del pensamiento situado o de ontologías relacionales para abordar la complejidad contemporánea. El pensamiento contemporáneo que duda de la realidad medible, evaluable e inmutable, que huye de los binarismos 
y las categorías encaja perfectamente con la idea de complejidad. En ella orbitan conceptos como el cambio, las relaciones y los sistemas que dependen del entorno o de las interacciones entre individuos: ideas que recogen las ontologías relacionales. La complejidad también requiere la observación de aquello que emerge como complejo, como veremos en el siguiente apartado, y esta idea de situar al observador siempre en el experimento o ejercicio, que evita hablar de objetividad o subjetividad nos acerca al pensamiento situado.

\section{3.- Complejidad}

No existe un consenso sobre la definición de complejidad. Hay varias aproximaciones desde las diferentes ramas del conocimiento que la utilizan: matemáticas, antropología, economía, urbanismo, física, sociología y biología entre otras. La justificación de cada una de estas aproximaciones radica menudo en su capacidad de producir valores intuitivamente correctos dentro del contexto en que se encuentra (Sporns, 2007).

El estudio de la complejidad y de los sistemas complejos analiza cómo un sistema formado por varios elementos exhibe un comportamiento emergente $^{2}$ que no es trivial.

Veamos como ejemplo las formas y coreografías de las bandadas de pájaros que se conforman, sin director alguno, siguiendo dos reglas bien sencillas: cada pájaro debe volar siguiendo a sus vecinos más cercanos y manteniendo cierta distancia con estos para evitar chocar.

Este comportamiento de flocking de los pájaros ha inspirado a varios artistas a explorar este sistema como, por ejemplo, el proyecto NOTbirds (Suárez, n.d.) donde se crea una animación programada de bandadas de pájaros que se pueden alterar en tiempo real, ver la imagen 1 y 2 .

\footnotetext{
${ }^{2}$ Se considera un fenómeno emergente aquel comportamiento macroscópico que no se puede predecir a partir del conocimiento de los elementos/individuos del sistema y sus reglas de funcionamiento. Para más detalles y discusión sobre el concepto de emergencia ver Soler (2019).
} 

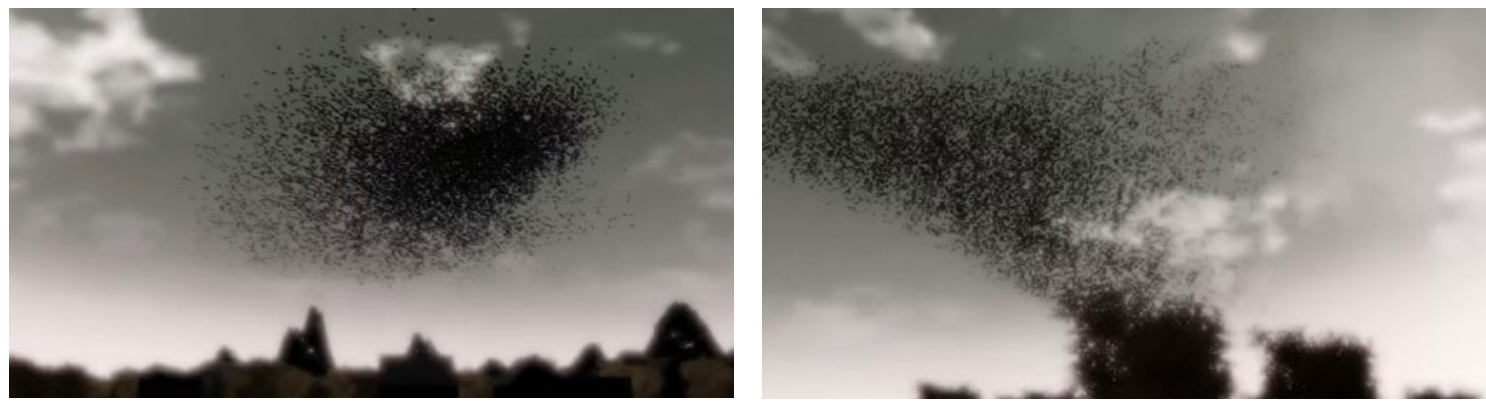

Imagen 1 y 2 - Instantes del vídeo del proyecto NOTbirds de Diego Suárez que muestran formas emergentes en el volar de bandadas de pájaros. Imágenes cedidas por el autor.

La naturaleza y la arquitectura de los sistemas complejos disponen que "dadas las propiedades de las partes y las leyes de su interacción, no es una cuestión trivial inferir las propiedades del todo" (Simon, 1981 p184). Y esta dificultad cuestiona la idea prevalente durante tiempo de que la percepción de la complejidad surge de la falta de información o del desconocimiento del comportamiento de un sistema donde la presencia de un gran número de variables y parámetros pueden enmascarar las regularidades subyacentes. Datos experimentales y avances teóricos han demostrado lo contrario, que la complejidad está arraigada en el comportamiento de ciertos sistemas (Nicolis y Rouvas-Nicolis, 2007). Es un concepto ubicuo en todo tipo de campos y hay un amplio abanico de propuestas de medida con el fin de aproximarse o acotar el grado de complejidad de un sistema o proceso. A pesar de los planteamientos heterogéneos para definir o medir la complejidad, se ha llegado al consenso de que hay ciertas características y propiedades comunes que definen todos los sistemas complejos (Gell-Mann, 1988; Mitchell, 2009; De Domenico et al., 2019).

Un sistema complejo:

Está formado por individuos que siguen reglas simples sin control central o liderazgo evidente. Esta característica se denomina auto-organización. Por ejemplo, las hormigas que construyen puentes para alcanzar la comida sin una directora evidente, ver la imagen 3 y 4.

Produce y usa información entre individuos que interactúan localmente entre sí a pequeña escala y, a veces, con el entorno. Por ejemplo, billones de neuronas interactuando en el cerebro. 
Cambia su estado macroscópico dinámicamente. Cambios en el tiempo, de forma no lineal, dan lugar a fenómenos impredecibles. Por ejemplo, la variación de la meteorología.

Puede variar su comportamiento y evolucionar. En este caso se llama sistema adaptativo. Por ejemplo, un sistema inmunológico aprende sobre nuevos patógenos.
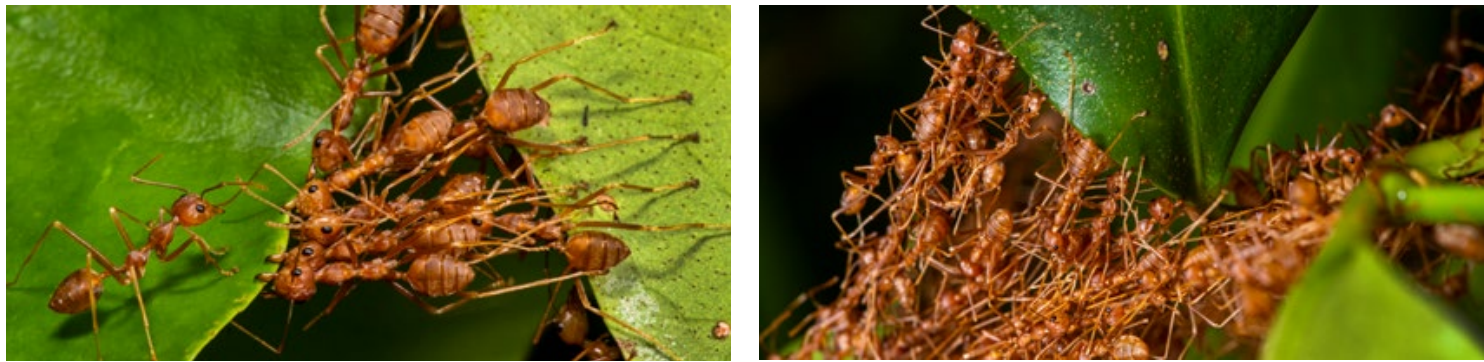

Imagen 3 y 4 - Fotos de Mistry (n.d.) para el proyecto Breathing Pixels que muestran hormigas auto-organizándose para crear puentes. Imágenes cedidas por el autor.

\section{1.- Los individuos de los sistemas complejos}

Un sistema complejo está formado por elementos, individuos, que son diferentes y funcionan como unidades del sistema. Individuos que siguen reglas de comportamiento concretas y generan una dinámica o comportamiento local, a menudo sin una dirección central que rija su conducta, por lo que forman sistemas auto-organizados, no jerárquicos, descentralizados, a los que podemos calificar como sistemas rizomáticos ${ }^{3}$.

Los elementos de un sistema complejo se pueden descomponer, un cierto número de veces, en otras unidades, dando lugar a sistemas complejos formados por sistemas complejos, creando estratos con varios niveles de organización.

Ya que la complejidad es un término interdisciplinario que aparece en muchas disciplinas y campos, en esta investigación se usa la palabra individuo para referirse a las unidades del sistema cuando éste evoca,

\footnotetext{
${ }^{3}$ Según Deleuze y Guattari (1980, p.22) los sistemas rizomaticos descentralizados son "redes de autómatas finitos en los que la comunicación se produce entre dos vecinos cualesquiera, en los que los tallos o canales no preexisten, en los que los individuos son todos intercambiables, definiéndose únicamente por un estado en un momento determinado, de tal manera que las operaciones locales se coordinan y que el resultado final global se sincroniza independientemente de una instancia central”.
} 
se inspira o trata temas sociales. También se usa la palabra individuo en la descripción de sistemas inspirados en comportamientos animales colectivos, como las bandadas de pájaros o los bancos de peces. Se usa la palabra elemento del sistema para referirse a unidades de sistemas más abstractos o que no se relacionan con ningún fenómeno sociológico de cualquier especie.

\section{2.- La información y las interacciones en los sistemas complejos}

En un sistema complejo los elementos interactúan localmente entre sí a pequeña escala $y$, a veces, con el entorno. Los individuos del sistema crean interacciones, produciendo e intercambiando información. Las interacciones suelen modular las acciones individuales alterando el comportamiento local de los individuos según sus vecinos, el entorno o el contexto global. En muchos sistemas complejos las interacciones entre subconjuntos de individuos están mediadas por alguna comunicación o conexión, ya sea una comunicación química como en las colonias de hormigas o bien una conexión electrónica como en las redes de Internet.

La complejidad aparece de manera muy destacada en sistemas que combinan elementos heterogéneos con interacción a gran escala. Estos sistemas se vuelven más complejos a medida que integran de manera más eficiente la información, es decir, que son más capaces de acoger la existencia de elementos especializados y la existencia de interacciones estructuradas que unen estos elementos en un todo coherente.

En sistemas simples, las propiedades del conjunto se pueden entender a partir de la agregación de sus elementos. Los comportamientos y propiedades macroscópicas de un sistema simple se pueden deducir de las propiedades microscópicas de sus partes.

Por el contrario, en un sistema complejo, emergen, aparecen, rasgos que abarcan el sistema en general y que no son triviales. Las interacciones entre elementos generan fenómenos o efectos que no se pueden deducir a partir de los comportamientos microscópicos de los elementos. Estos fenómenos, comportamientos macroscópicos, surgen como resultado de las múltiples relaciones y son una propiedad del conjunto de todo el sistema. En muchos casos, incluso un examen detallado y completo de los elementos individuales y sus interacciones no permite 
predecir la gama de procesos emergentes. La interacción entre individuos del sistema genera y exhibe estructuras y conductas colectivas no triviales a gran escala.

Las propiedades emergentes, por tanto, reflejan el papel primordial de las interacciones entre individuos. Se manifiestan mediante la creación de estados auto-organizados de tipo modular y de generación de novedad (Soler, 2019), donde la coherencia se asegura y se establece mediante un mecanismo de abajo hacia a arriba en lugar de provenir de un diseño o un control de arriba hacia abajo.

\section{3.- La dinámica de los sistemas complejos}

Un sistema complejo tiende a cambiar su estado macroscópico dinámicamente, cambios a lo largo del tiempo, de forma no lineal, dando lugar a fenómenos impredecibles, a menudo, con multiplicidad de resultados posibles. Por ejemplo, un pájaro volando y adaptándose a las corrientes de aire.

Mirar los sistemas de forma dinámica es analizar los cambios a lo largo del tiempo. Un estado, un instante, describe las características del sistema en ese momento, una especie de foto fija. A medida que el sistema cambia de estado, sus características también cambian. Este cambio se llama lineal si es directamente proporcional al estado actual, al tiempo y a la variación del entorno. Y se llama cambio no lineal si no es proporcional. Los sistemas complejos suelen ser no lineales ${ }^{4} y$ evolucionan, cambian, de forma muy difícil de predecir.

\section{4.- El cambio en los sistemas complejos}

Cuando un elemento del sistema está dañado o desaparece, a menudo los sistemas complejos pueden recuperar su funcionalidad anterior e incluso mejorarla: se adaptan, varían su comportamiento y evolucionan. Esto se logra mediante la robustez, la capacidad de soportar perturbaciones y cambios; mediante la resiliencia, la capacidad de volver al estado original después de una gran perturbación; o mediante la adaptación, la capacidad de cambiar el sistema para mantenerlo funcional y sobrevivir.

\footnotetext{
${ }^{4}$ Esta afirmación parece indicar que un sistema que no sea lineal será difícil de predecir. Sin embargo, no siempre es así. Hay sistemas no lineales, por ejemplo, los eclipses lunares o solares, que sabemos predecir perfectamente y determinar cuando ocurrirán.
} 


\section{4.- Pensamiento sistémico y pensamiento contemporáneo aplicado a la complejidad}

Una vez acotada la complejidad y presentadas las características de los sistemas complejos podemos relacionarlo con el pensamiento sistémico. Como menciona Rowan (2020) "seguimos operando con esquemas de la comprensión de la realidad modernos cuando los problemas a los que nos enfrentamos son cada vez más complejos”. Veamos como el pensamiento sistémico nos ofrece un marco para abandonar ciertas ideas de la modernidad a la vez que presenta un encaje perfecto con algunas características intrínsecas de la complejidad.

De la estructura al proceso: el pensamiento moderno plantea la existencia de substancias, estructuras fundamentales y/o bloques esenciales; y de ciertas fuerzas y mecanismos que hacen que estas estructuras interactúen, dando lugar a ciertos procesos. En el marco del pensamiento sistémico cada estructura se considera una manifestación del proceso subyacente. Este enfoque se encuentra con la idea contemporánea de Deleuze y Guattari (1980) de que el ser ya no es una esencia, una substancia, sino un devenir, un proceso, una presencia.

De lo estático a lo dinámico: el pensamiento moderno propone certezas fijas; certezas medibles, acotables y fijables. Construir o alterar un sistema permite ver como se comporta, como son sus fluctuaciones, explorar y entender su pasado, su historia, estudiar sus cambios. Observar el comportamiento de un sistema obliga a centrarse en los hechos que ocurren en ese sistema, olvidando teorías, medidas, ideas preconcebidas o prejuicios. Centrarse en lo que sucede dirige el pensamiento y la investigación a ideas y conceptos dinámicos en vez de estáticos (Meadows, 2009).

De la parte al todo: el pensamiento moderno cree que en todo sistema complejo la dinámica del todo puede ser entendida a partir de la comprensión de las partes. Y estas partes no pueden ser analizadas más allá de reducirlas a partes más pequeñas. En el marco del pensamiento sistémico, las relaciones 
de los elementos y su comportamiento microscópico no determina el comportamiento del todo, el comportamiento macroscópico global. Se focaliza en la observación de este todo, del sistema y de aquello que emerge para preguntarse por las relaciones y huir de razonamientos de causa-efecto.

De la "verdad" fenomenológica a las aproximaciones: el pensamiento sistémico presenta la realidad como una red de relaciones dinámicas e interconectadas, donde identificar ciertos patrones o comportamientos depende del observador. Puesto que el observador influye en los resultados del experimento, idea tomada de la cibernética, se entiende que el observador siempre se tiene que situar en el experimento o ejercicio. Es la respuesta contemporánea del pensamiento situado (Barad, 2007). No se puede proveer nunca de un saber completo y definitivo, no se presenta la verdad como una correspondencia precisa entre la descripción y el fenómeno. Solamente se puede plantear como una visión limitada y situada de la realidad abriendo la puerta a la inclusión y exploración de nuevos saberes y epistemologías del sur. Como por ejemplo en el proyecto Khipu que utiliza la técnica inca del registro de información por nudos, los khipus, juntamente con electrónica digital para codificar datos lógicos y dotar de cierta algoritmia a una computadora textil (Piña, 2017).

Del resultado a las relaciones e interconexiones: el sistema crea su propio comportamiento a partir de las interacciones internas y de ciertas influencias externas. Los eventos externos a veces no se pueden controlar, las interacciones internas se pueden moldear. Se dirige la atención a las relaciones e interconexiones en vez de al resultado final global recogiendo la idea de las ontologías relacionales cuando proponen que los resultados dependen de estas relaciones, e incluso que las cosas no preexisten a sus relaciones (Haraway, 1995) y nos instan a ver interdependencias, vínculos y relaciones donde antes solo veíamos límites entre especies, sujetos y objetos (Haraway, 2016; Puig de la Bellcasa, 2017). 


\section{5.- Arte generativo}

Las anteriores ideas y aproximaciones pueden ser exploradas de forma práctica a través del diseño o del arte, concretamente del arte generativo definido por Galanter (2003) como: "la práctica artística que usa sistemas -máquinas, código informático, instrucciones procedimentales o un conjunto de reglas- que se ponen en funcionamiento con un cierto grado de autonomía y que resultan en una pieza artística”.

Esta investigación acota los proyectos de arte generativo a aquellos que usan código informático para su realización y que caen dentro del amplio campo del arte digital. Proyectos y piezas que se centran en usar el código y las instrucciones como método fundamental y sustrato de creación de la pieza artística -arte por computador, computer art-en contraposición a otros proyectos de arte digital que usan los ordenadores como herramienta para la creación -arte asistido por computador, computer assisted art-.

Siguiendo esta dicotomía, establecida por Boden y Edmonds (2009), y su taxonomía de arte digital, tampoco se ajustan a esta investigación proyectos de arte digital que pueden incluir algún subsistema generativo, pero donde éste no es el eje central de la propuesta artística como son el arte evolutivo, el arte electrónico, el arte con robots o la realidad virtual. Tampoco se incluyen en este estudio, puesto que no se trata de sistemas con cierta complejidad, el arte abstracto basado en la permutación y la serialidad, típico de los años 60 del s. XX.

El arte generativo permite crear sistemas dinámicos y ponerlos en funcionamiento o detenerlos; ver la evolución de todo el proceso; observar el resultado visual y ver si muestra algún tipo de fenómeno emergente; alterar pequeños detalles y explorar su complejidad. Piezas gráficas animadas, que se centran en las propiedades emergentes de un sistema formalizado a partir de algoritmos. Sistemas programados con código informático del cual se pueden modificar las relaciones, las dinámicas, los comportamientos de los individuos, sus interacciones y/o las condiciones iniciales del sistema. Son piezas que recogen todas las características de los sistemas complejos enumeradas en el apartado 3. 
El artista o diseñador usa un ordenador como herramienta y establece instrucciones concretas y reglas escritas. Un lenguaje de programación, con su vocabulario acotado, semántica rígida y estructuras fijas que la computadora debe poder interpretar sin ambigüedad. ¿Cómo puede una práctica tan estricta y acotada generar sorpresa, resultados impredecibles o promover la aparición de lo inesperado?

Si un diseñador especifica los objetivos de su diseño, sus partes y todos los comportamientos de estas partes constituyentes, el eventual funcionamiento de ese diseño y el resultado estarán limitados por lo que el diseñador pueda imaginar. El sistema estará cerrado a la novedad y solo responde a ideas preconcebidas que se han integrado explícita o implícitamente en él.

En cambio, al tratarse de piezas con intenciones artísticas no se pretende hacer una simulación o demostrar ningún fenómeno. El artista decide y controla la construcción del sistema, sin embargo, este fluye y deriva en comportamientos y resultados que no están totalmente bajo su control, aunque sí están acotados y moldeados por el artista. Este proceso de trabajo dota de gran libertad de exploración y el proceso de creación permite al artista tomar nuevas direcciones a partir de lo que observa y sucede, incluso si ha llegado a ello por error. Algunos artistas generativos, como Hoff $(2017 ; 2018)$ o Hobbs (2014) han escrito sobre sus procesos de creación y como el código de programación permite la sorpresa y la exploración de comportamientos y resultados emergentes. Una idea inicial de la pieza que quieren dibujar les sirve como semilla para empezar a construir el sistema que la creará. Sin embargo, en cierto momento algún error, algún desajuste numérico, error tipográfico o alguna corrupción de los datos, revela visualmente un resultado inesperado. Estos fallos, llamados también bugs, son habituales en el código de programación y desencadenan resultados indeseados que alimentan la inspiración del artista. Este abandona la idea inicial para trabajar a partir del nuevo sistema en una nueva dirección. Solamente la práctica artística de arte generativo permite la libertad de aprovechar los fallos de código. La creación de código para software funcional, por el contrario, debe eliminar esos errores con tal de obtener el resultado diseñado previamente. 
Eno (1976) ha discutido también el uso creativo de sistemas generativos en la práctica artística musical, que, como en nuestro caso, se alejan de simulaciones, de cálculos o de objetivos prácticos. Su propuesta, se extiende aquí a la práctica del arte generativo, para proponer que estos proyectos usan heurísticas, instrucciones explorativas, en contraposición al uso de algoritmos, instrucciones que buscan un objetivo concreto $^{5}$.

El arte generativo que plantea un enfoque explorativo, instrucciones heurísticas y que acepta el error dentro del flujo de trabajo, encaja perfectamente como práctica para explorar la complejidad y todas las ideas que la acompañan y que destila.

\section{6.- Complejidad en los sistemas generativos}

En la naturaleza, y en el arte generativo, lo complejo se encuentra a medio camino entre el orden (por ejemplo, en un cristal) y el desorden (por ejemplo, en un gas) (Solé y Manrubia, 2001). A nivel más formal las piezas gráficas generativas se pueden situar en un continuo, mostrado en la fig. 1 y formalizado por Galanter (2003). Las propuestas van de los sistemas ordenados, que contienen poca información y que

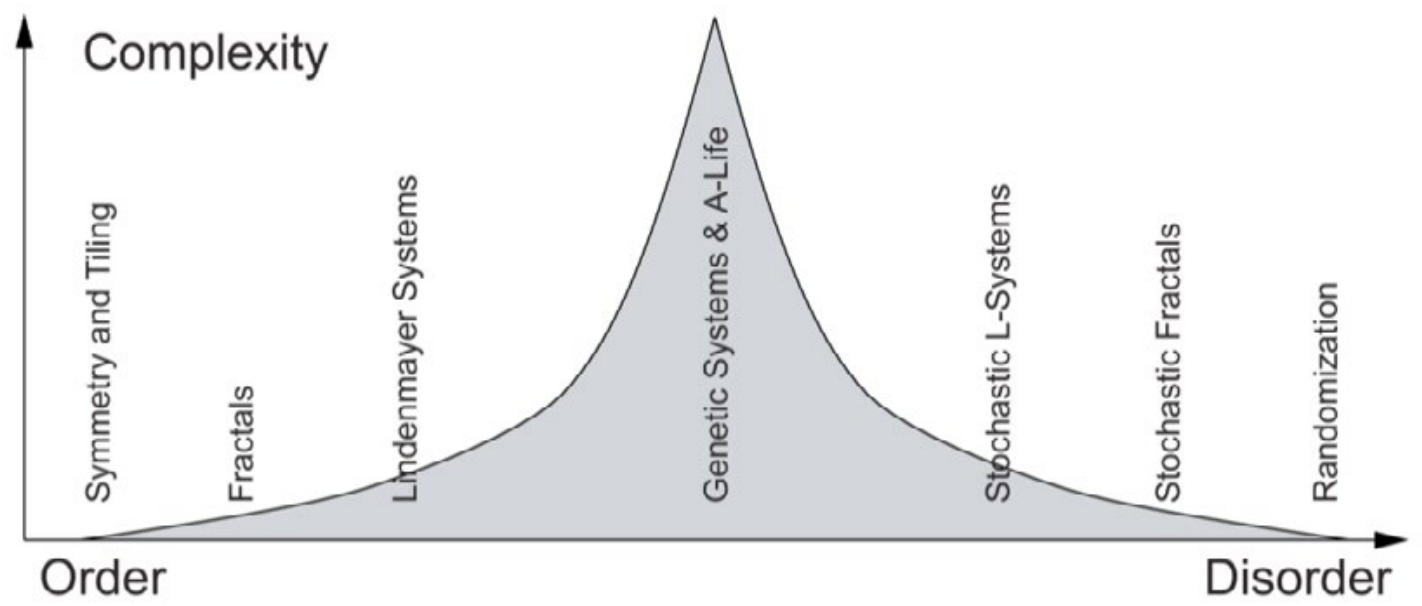

Fig. 1 - Complejidad de las diversos sistemas gráficos generativos en un continuo entre orden y desorden (Galanter, 2003).

\footnotetext{
${ }^{5}$ Según la definición de Stafford Beer citada por Eno (1976, p232) en la práctica artística generativa la heurística se refiere al "conjunto de instrucciones para alcanzar un objetivo desconocido por exploración, que evalúa continuamente su progreso según algún criterio conocido” mientras que los algoritmos son el "conjunto de instrucciones para alcanzar un objetivo concreto".
} 
son de comprensión alta por parte del público; a los sistemas caóticos, que contienen gran cantidad de información pero que son de baja comprensión debido a su desorden y falta de redundancia.

Se usan conceptos de computación y teoría de la información para medir o caracterizar orden y desorden, simplicidad y complejidad (Sporns, 2007; Mitchell, 2009; Crutchfield, 2012). El conocimiento del proceso algorítmico interno, del código informático, y el resultado que se genera en la pieza gráfica, permite desde el marco del arte generativo acercarse a algunas de estas medidas y caracterizaciones.

Las medidas de complejidad se pueden separar en dos grupos. Dentro del primero, están las medidas que capturan la aleatoriedad, el contenido de información o la longitud de la descripción del sistema. Dentro de este grupo los comportamientos aleatorios son los que tienen la máxima complejidad.

El segundo grupo conceptualiza la complejidad de forma diferente al azar: en este grupo los sistemas complejos son aquellos que poseen una gran cantidad de estructura o información, a menudo a varias escalas temporales y espaciales. Dentro de este grupo de medidas, los sistemas altamente complejos se sitúan en algún lugar entre sistemas ordenados -regulares-o desordenados -aleatorios-. Es esta la caracterización que corresponde a las piezas de arte generativo.

No nos atañe medir exactamente la complejidad de las piezas, sin embargo, es interesante acotar y caracterizar en qué espacio aparece la complejidad. Todo ello con el objetivo de proponer, conceptualizar y crear piezas generativas complejas en las que podremos explorar y extrapolar todo lo discutido sobre estos sistemas, véase la imagen 5.

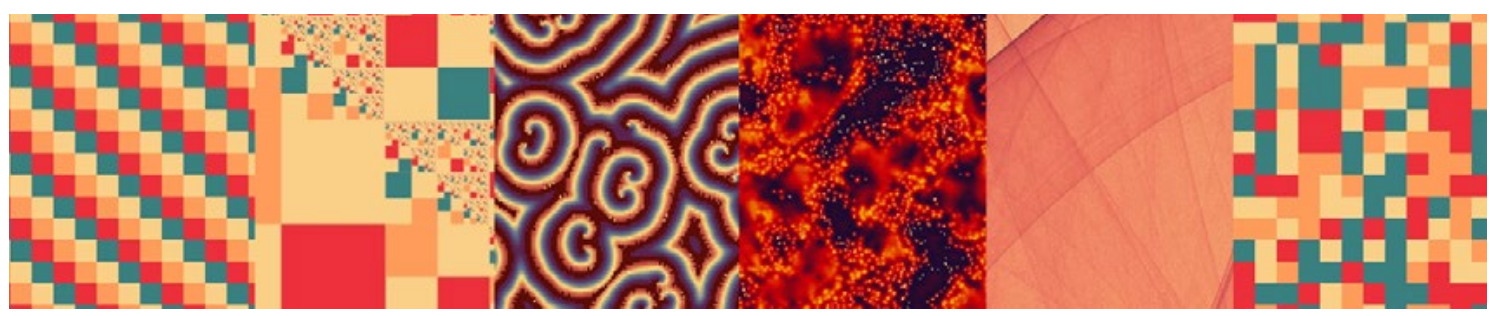

Imagen 5 - Dibujos generados, de izquierda a derecha: repetición y patrones ordenados, fractales, dos sistemas de autómatas celulares, atractores y aleatoriedad total. 


\section{7.- La práctica}

Con el título Quarantena Generativa arranca un proyecto durante el confinamiento por Covid-19 que plantea a largo plazo recoger piezas de arte generativo creadas con la finalidad de explorar la complejidad y las ideas planteadas en este artículo (Carreras, 2020). Son piezas creadas en uno o dos días a modo de experimentaciones. Son bocetos, prototipos de alguna exploración visual o piezas más acabadas y completas.

Por ejemplo, las imágenes 6,7,8 y 9 muestran varias versiones de una investigación visual de la secuencia de Recamán que indaga en la recurrencia y relación entre sus elementos. Enlazando los valores numéricos de esta secuencia con arcos redondeados se crean patrones de ida y vuelta. Dibujando los enlaces con curvas más deformadas, se consigue un resultado final que parece cierta escritura a mano hecha de forma rápida.
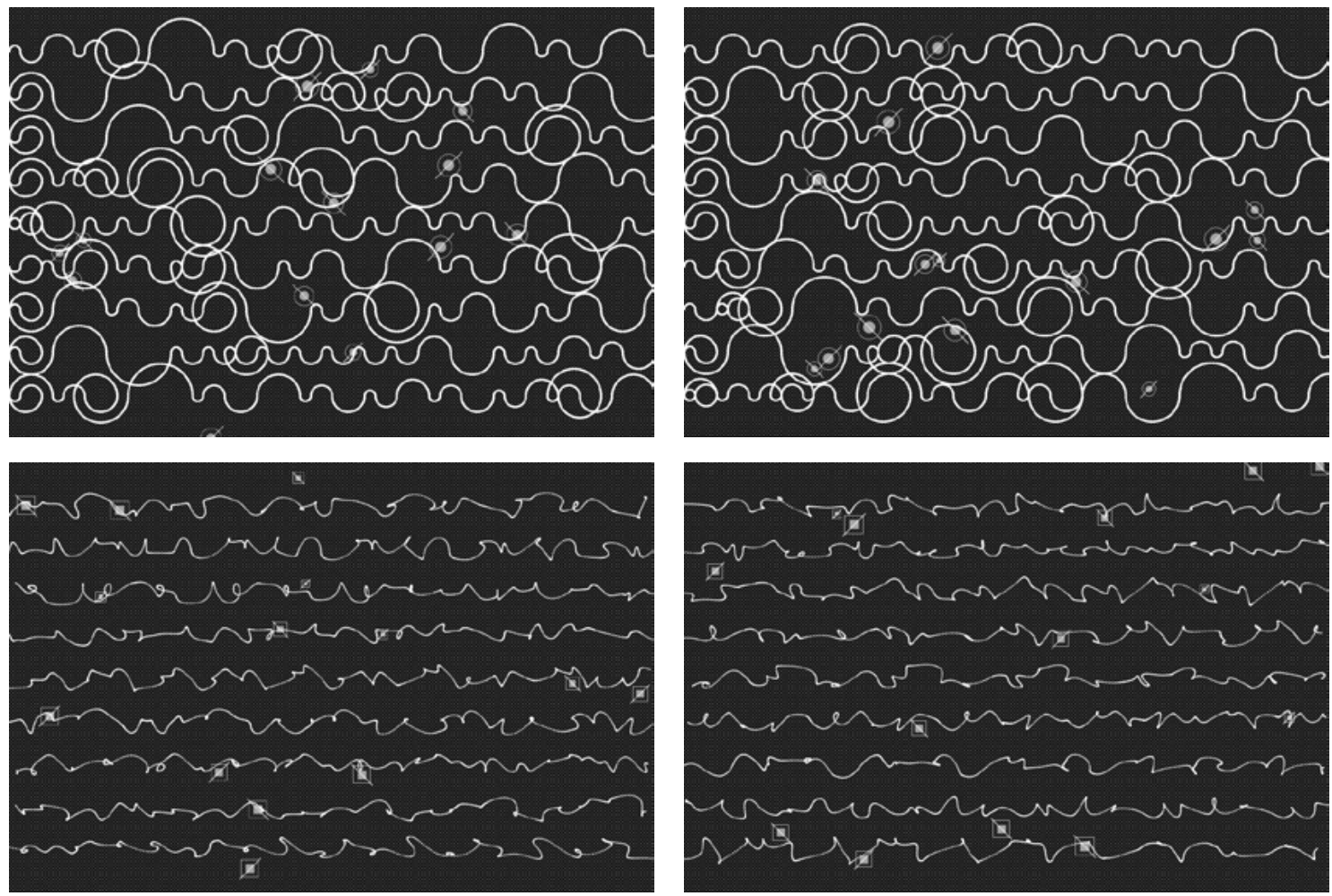

Imágenes 6, 7, 8 y 9 - Propuestas visuales generadas a partir de la secuencia de Recamán que exploran la recurrencia y relación entre sus elementos. 
Otras propuestas exploran instantáneas de la evolución de un sistema en vez de variaciones del mismo. Las imágenes $10,11,12$ y 13 son instantes de una pieza en que se forman estructuras burbujeantes a partir de la mezcla desordenada de colores.
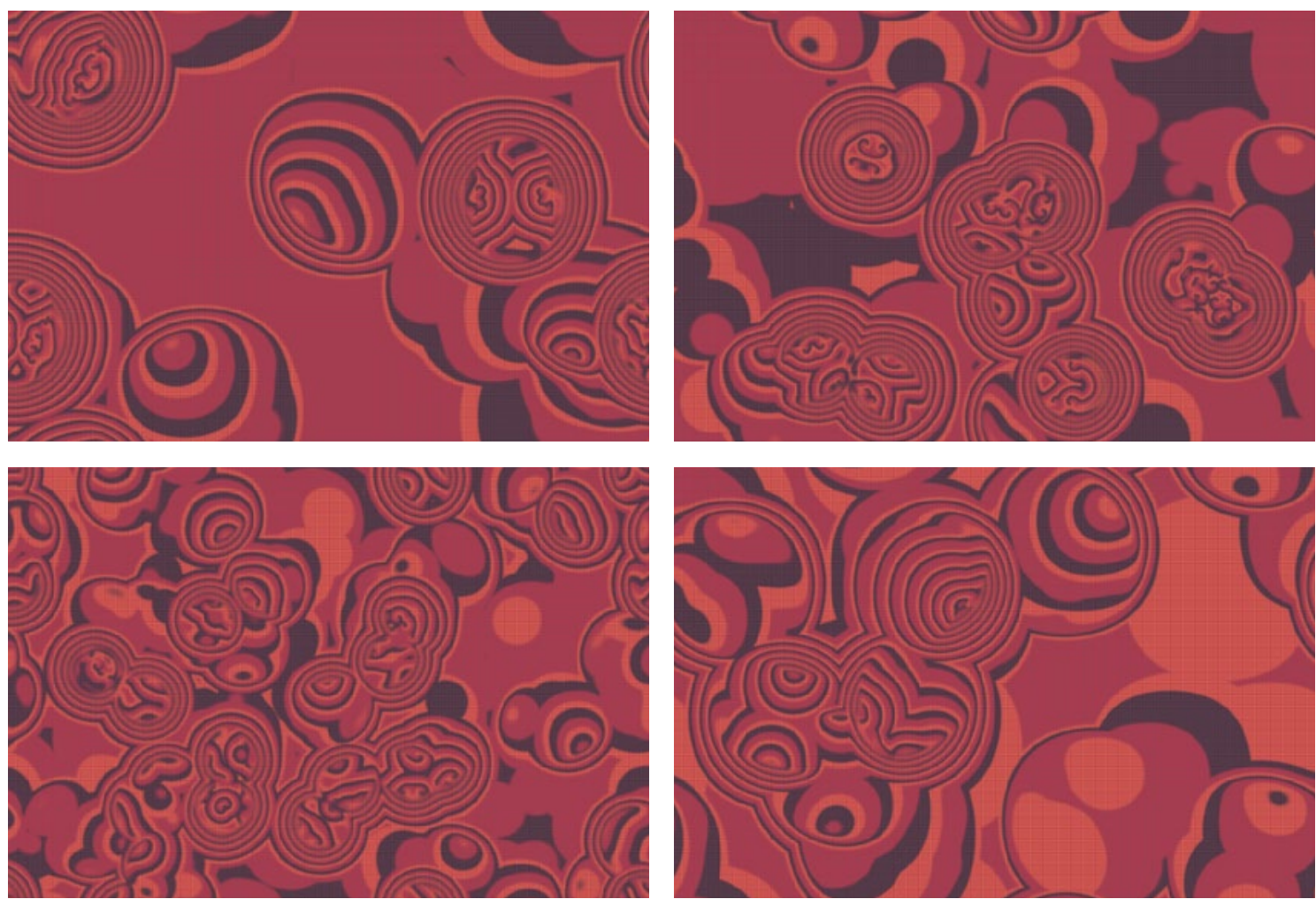

Imágenes 10, 11, 12 y 13 - Simulación de la reacción BZ, reacción química que genera patrones oscilantes emergentes. 
Otras piezas, como la que se muestra a continuación, explora la dinámica del sistema, los cambios en cada iteración y la emergencia de formas, imágenes 14, 15, 16, 17 y 18. Este sistema plástico de franjas ruidosas horizontales se encapsula en una zona circular en la que crecen y aparecen formas que sugieren anillos de crecimiento de plantas arbóreas o se parecen a membranas celulares. Incluso en la imagen 18 aparece una pequeña forma de corazón que no se ha dibujado directamente sino que surge fruto de la dinámica del sistema.
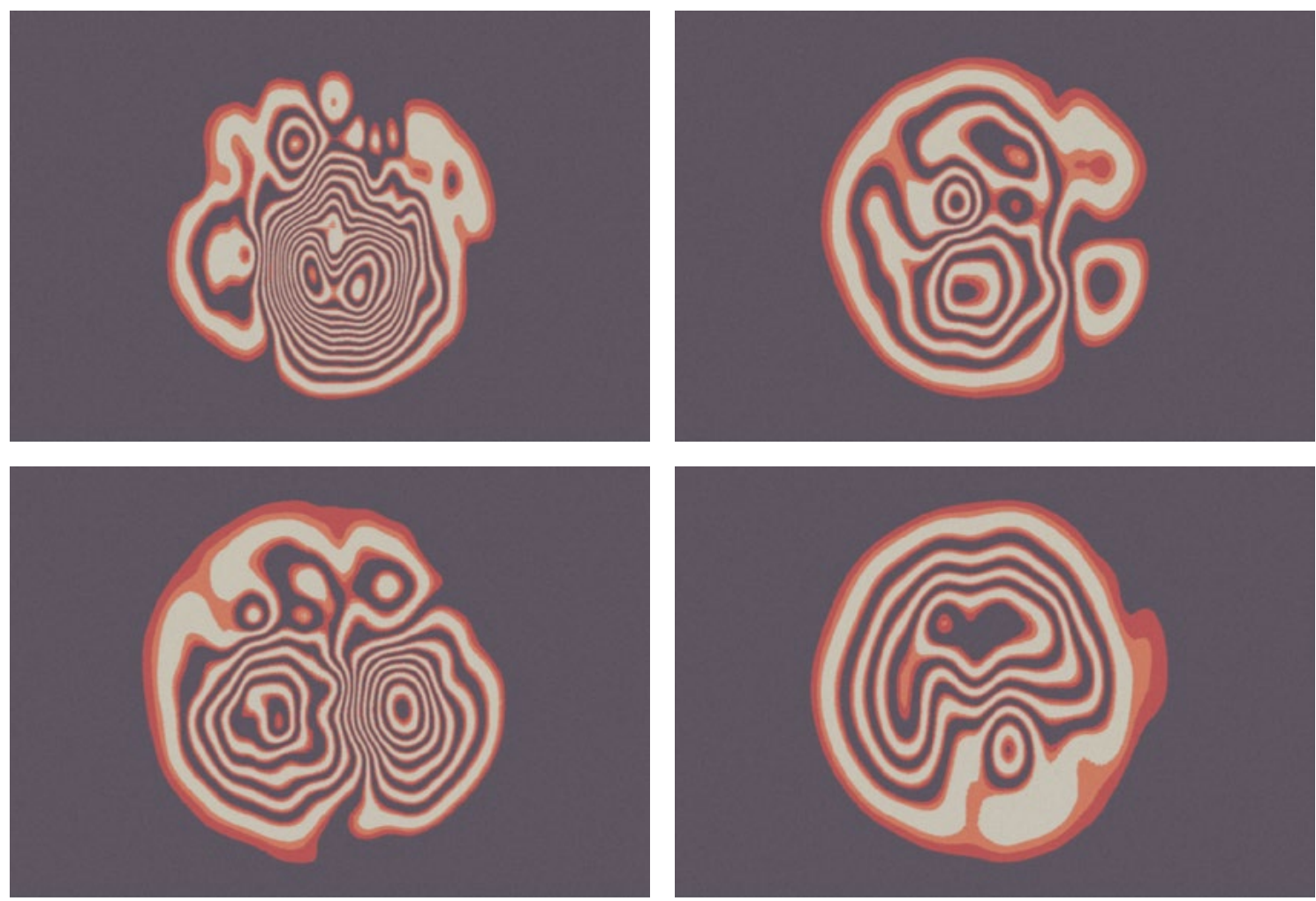

Imágenes 14, 15, 16, 17 y 18 - Sistema plástico de franjas encapsulado en un círculo en diversas iteraciones de su evolución. 
Hay piezas que arrancan de cero y otras que son evoluciones de otras piezas de días anteriores o de ideas anteriores. Por ejemplo, las estructuras tubulares que sugieren conexiones neuronales o fibras, que muestra la imágen 19. Aparecen, se forman, a partir de manchas líquidas, metaballs, que fluyen por el espacio, mezclandose, y a las que dejamos pintar su camino.
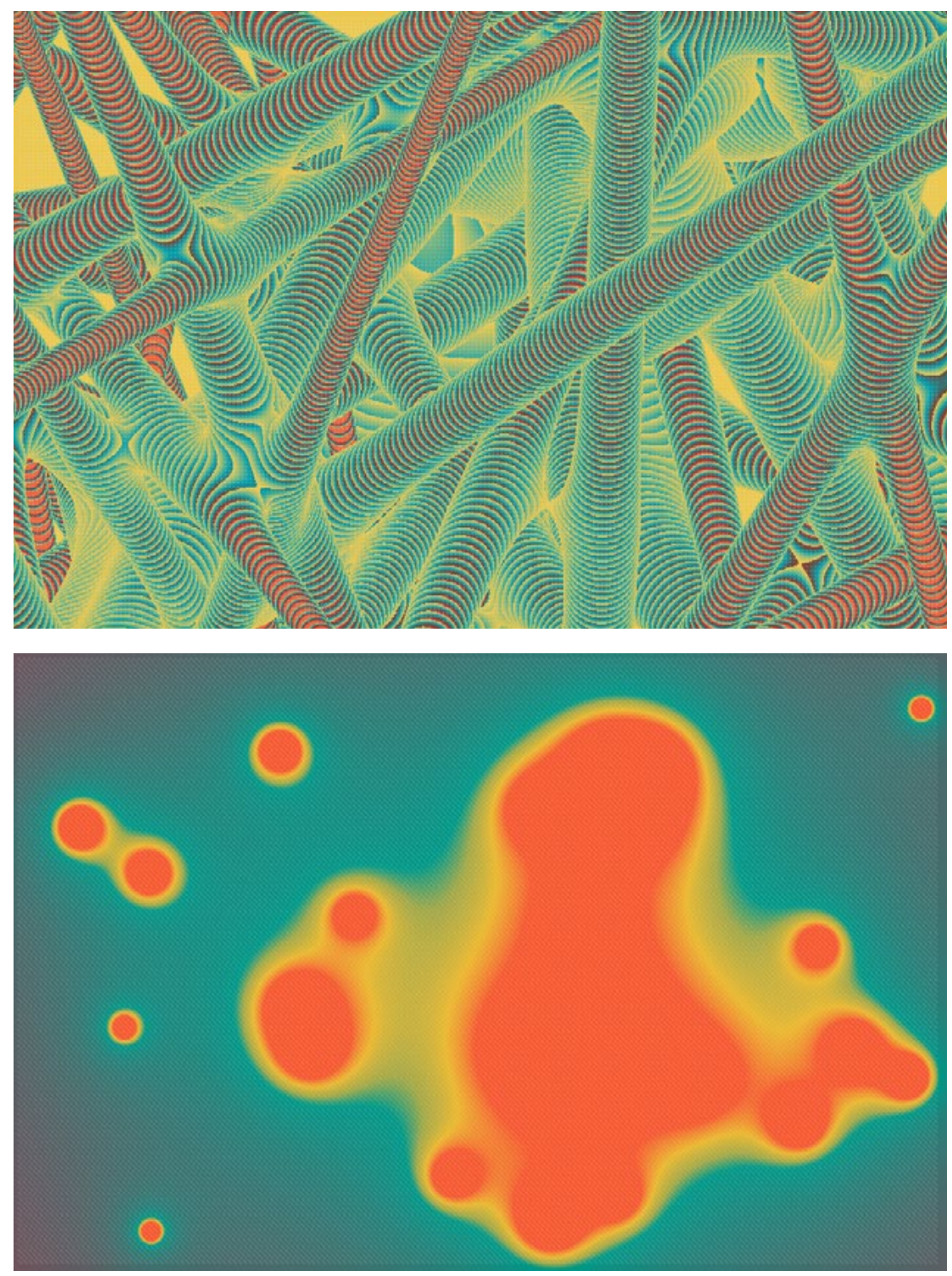

Imagen 19 y 20 - Estructuras tubulares nacidas de la unión plástica de círculos en movimiento por el espacio que se aglomeran al encontrarse formando manchas. 
Y, finalmente, hay algunas piezas del proyecto Quarantena Generativa que exploran los extremos de la complejidad generativa apostando por un orden o desorden evidente, imágenes 21, 22, 23 y 24.
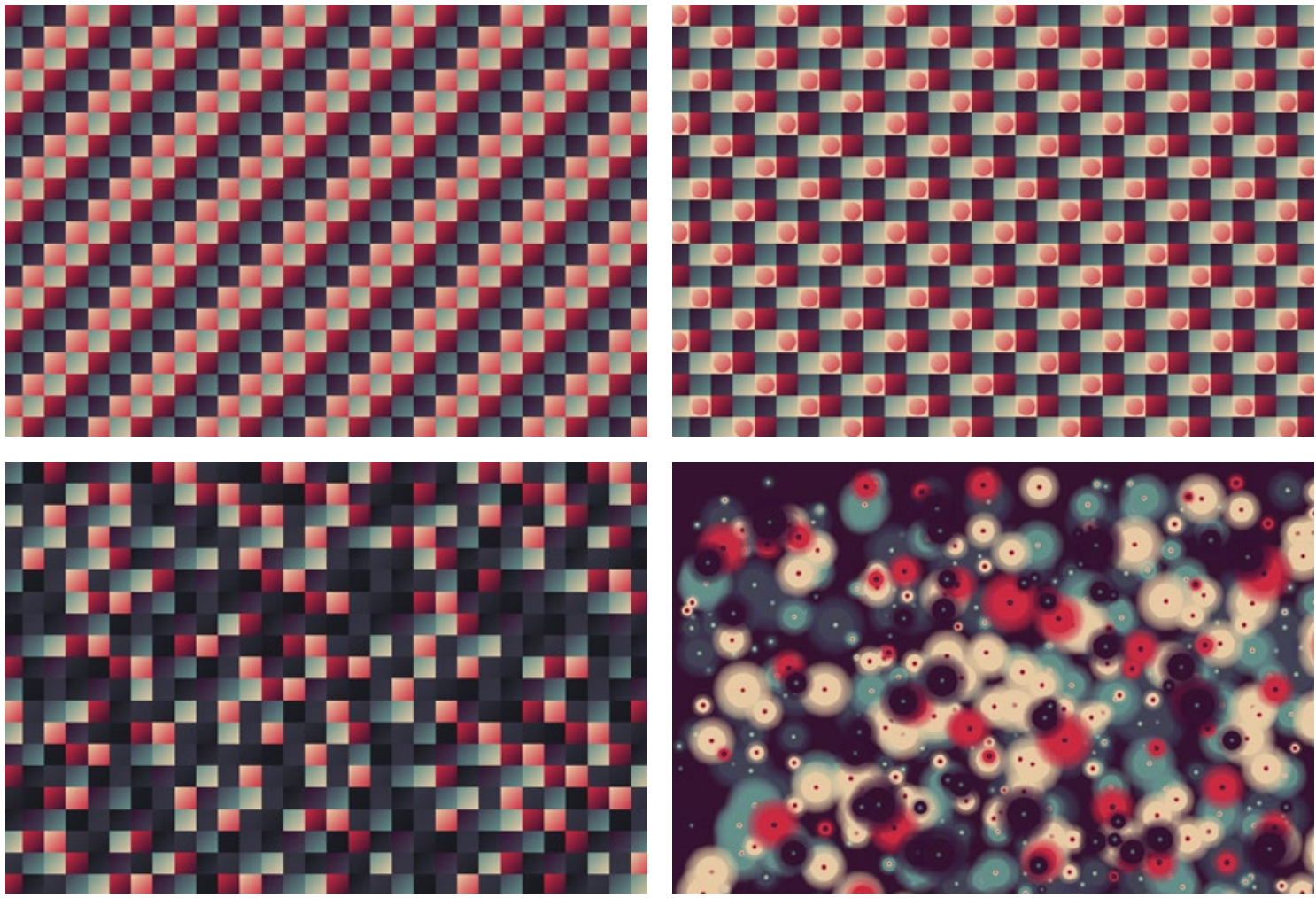

Imágenes 21, 22, 23 y 24 - Exploraciones de patrones de repetición ordenados y desorden absoluto. 
Para dotar de cierto desorden las piezas gráficas generativas se usan números aleatorios y ruido. Los números elegidos al azar introducen variaciones en el resultado gráfico. Las formas, los colores elegidos y los comportamientos creados por este azar pueden parecer a menudo caóticos. El azar y el caos son indistinguibles a nivel gráfico ya que se desconoce el sistema y las leyes que rigen lo que observamos a nivel macroscópico. En el contexto de los sistemas de arte generativo, azar y caos a veces son indistinguibles. Sin embargo, la aleatoriedad no es caótica y el caos puede aparecer sin aleatoriedad. Más azar, más aleatoriedad o ruido, añaden más variedad a las piezas gráficas, pero esto no implica más complejidad. La diversidad y la variedad pueden emerger del propio sistema, como en el siguiente ejemplo en que círculos crecientes nacen de un punto y se ramifican según la dinámica de crecimiento establecida en la heurística, ver las imágenes 25, 26 y 27.
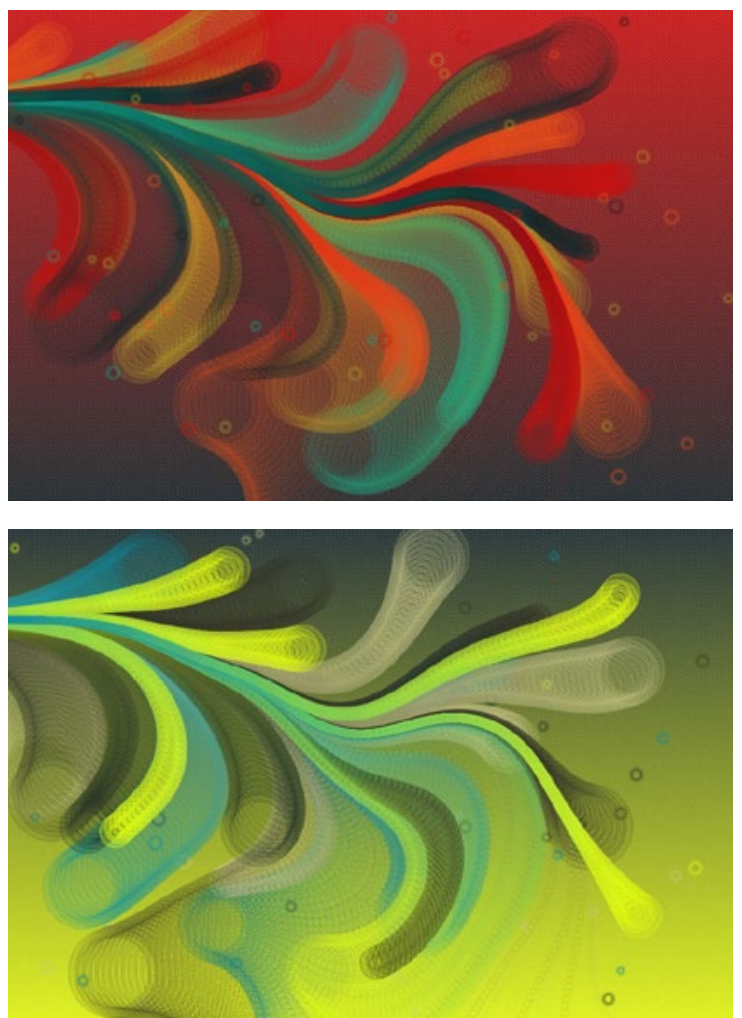

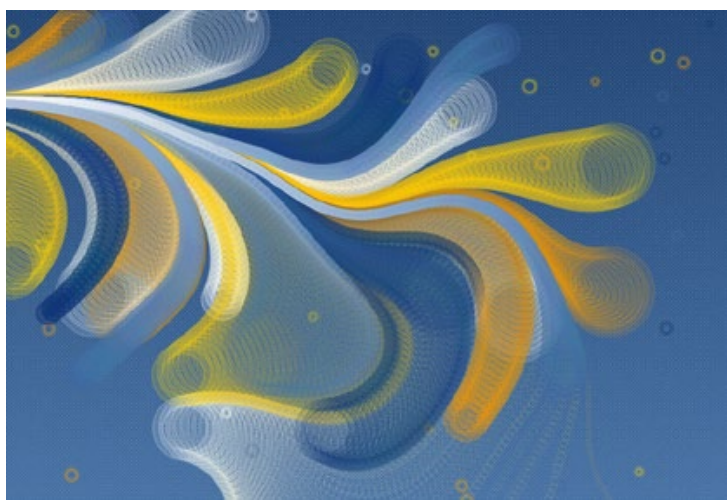

Imágenes 25, 26 y 27 - Sistema de ramificación y crecimiento. 


\section{8.- Conclusiones}

Hemos presentado y discutido como la práctica artística del arte generativo, acompañada de propuestas ampliamente heurísticas y un pensamiento sistémico, permiten acercarnos a los sistemas complejos desde una perspectiva contemporánea: enfatizando los procesos y las relaciones, evidenciando el dinamismo y el cambio continuo de todo sistema, huyendo de binarismos y catalogaciones, aproximándonos con una mirada holística a unos sistemas que no se pueden domesticar y que nos ofrecen sorpresas emergentes.

Esta práctica no está exenta de interrogantes. ¿Es el hábito de construir sistemas con algoritmos y su sustancia estructurada la que conlleva inevitablemente a un pensamiento sistémico? ¿ $\mathrm{O}$ es la forma de mirar, entender, pensar y plantear como sistema todas las cosas, aquello que lleva al artista a desarrollar su práctica en forma de arte generativo? El eterno dilema del huevo o la gallina. Sea como fuere, este artículo pone de manifiesto la adecuación del arte generativo como práctica para explorar el comportamiento de sistemas. Si esta práctica y este marco permite atisbar alguna idea o dilucidar algún conocimiento que nos acerque a la complejidad será también una sorpresa.

En el proceso, se abren caminos para observar, explorar y profundizar en muchos de los conceptos que han aparecido a lo largo del texto, o bien en ideas que, sin nombrarlas, acompañan a la complejidad. Todo ello de la mano del pensamiento situado, visiones holísticas, dinámicas y rizomáticas, ontologías relacionales o nuevas epistemologías. Porque por pequeños o sencillos que parezcan, los sistemas complejos nos hablan de: individuos, elementos, comportamiento microscópico, comportamiento macroscópico, emergencia, auto-organización, no liderazgo, descentralización, relaciones, conexiones, interacción con el entorno, interacción entre individuos, información, diálogo, colaboración, evolución, adaptación, resiliencia, aprendizaje, cambio, supervivencia, orden, desorden, equilibrio, aleatoriedad, ruido, caos, diversidad e identidad. 


\section{Bibliografía}

Barad, K., 2007. Meeting the Universe Halfway: Quantum Physics and the Entanglement of Matter and Meaning. Durham. Duke University Press

Boden, M., Edmonds, E., 2009. What is generative art? Digital Creativity, 20, (1-2) pp.2146. ISSN 14626268

Autora, A., 2020. Quarantena Generativa. https://www.autora.com/quarantena-generativa [Accessed 5 diciembre 2021]

Crutchfield, J., 2012. Between order and chaos. Nature Phys, 8, (17-24).

De Domenico M., Brockmann D., Camargo C., Gershenson C., Goldsmith D., Jeschonnek S., Kay L., Nichele S., Nicolás J.R., Schmickl T., Stella M., Brandoff J., Martínez Salinas A.J., Sayama H., 2019. Complexity Explained. http://dx.doi.org/10.17605/OSF.IO/TQGNW

Deleuze G., Guattari F., 1980. Mil Mesetas. Capitalismo y Esquizofrenia. Valencia. Pre-textos.

Eno B., 1976. Generating and Organizing Variety in the Arts. In: Audio Culture Reading in Modern Music. Ed. Cox.C, Warner.D. London. Bloomsbury. pp 226-233.

Galanter, P., 2003. What is Generative Art? Complexity theory as a context for art theory. In: Proceedings of the International Conference on Generative Art. Milan. Italy.

Gell-Mann, M., 1988. Simplicity and Complexity in the Description of Nature. Engineering and Science, 51 (3) pp. 2-9. ISSN 0013-7812

Haraway, D. J., 1995. Ciencia, cyborgs y mujeres. La reinvención de la naturaleza. Madrid. Cátedra.

Haraway, D. J., 2016. Staying with the Trouble. Durham. Duke University Press.

Hoff A., 2017. A propensity for mistakes. https://inconvergent.net/2017/a-propensity-for-mistakes [Accessed 5 diciembre 2021]

Hoff A., 2018. Order and Chaos. https://inconvergent.net/2018/order-and-chaos [Accessed 5 diciembre 2021]

Hobbs T., 2014. Randomness in the composition of artwork. https://tylerxhobbs.com/essays/2014/randomness-in-the-composition-of-artwork [Accessed 5 diciembre 2021] 
Meadows, D. H., 2009. Thinking in Systems: A Primer. London. Stearling VA.

Mistry, U., n.d. Breathing pixels. http://earthcolab.com/portfolio/breathing-pixels [Accessed 5 diciembre 2021]

Mitchell M., 2009. Complexity: a guided tour. Oxford University Press. ISBN 0199798109

Nicolis, G., Rouvas-Nicolis, C., 2007. Complex systems. Scholarpedia 2(11):1473.

Paul, C., 2015. Digital Art. Thames and Hudson Ltd. UK. ISBN 0500204233.

Piña, C., 2017. Khipu: computación textil y sonificación de espectros.

https://proyectokhipu.wordpress.com [Accessed 5 diciembre 2021]

Puig de la Bellcasa, M., 2017. Matters of Care: Speculative Ethics in More than Human Worlds. University of Minnesota Press.

Rowan, J., 2020. Erótica, vínculos e interdependencia. Diseños de cuidado. Inmaterial. Diseño, Arte y Sociedad. 5 (9). pp 41-60. https://doi.org/10.46516/inmaterial.v5.81

Shanken, E., 2015. Systems. London and Cambridge MA: Whitechapel Gallery and MIT Press. ISBN 9780262527194.

Simon, H.A., 1981. The Sciences of the Artificial. MIT Press Cambridge.

Solé R., Manrubia S., 2001. Orden y caos en sistemas complejos. Politext Series 93-94. Edicions UPC. ISBN 8483014300, 9788483014301.

Soler J., 2019. The Open, the Closed and the Emergent: Theorizing Emergence for Videogame Studies. Journal of Game Studies, 19 (2) pp 1-17.

Sporns 0., 2007. Complexity. Scholarpedia, 2(10):1623.

Suárez, D., n.d. NOTbirds. http://diegosuba.com/portfolio/notbirds [Accessed 5 Diciembre 2021]

Wagensberg, J., 1998. Ideas sobre la complejidad del mundo. Tusquets Editores S.A. 
Anna Carreras, licenciada en Ingeniería de Telecomunicaciones por la Universidad Politécnica de Cataluña, UPC . Máster en Tecnologías Audiovisuales en la Universidad Pompeu Fabra, UPF. Artista digital. Programadora

Centra su práctica e investigación en los sistemas generativos y la interacción. Explora a escala audiovisual el orden y el caos en estos sistemas, equilibrio del que emergen comportamientos complejos. Su interés reside en emplear algoritmos, códigos y reglas para crear visuales que planteen lenguajes narrativos o alimenten reflexiones en la audiencia. Ha trabajado en empresas como Reactable Systems, Cuatic o Ping Pong Technologies y ha desarrollado como freelance proyectos para la Expo de Zaragoza, El Foro de las Culturas Barcelona 2004, el museo de Cidade da Cultura, Medialab Prado y Google Devart.

Profesora del Master en Innovación Audiovisual y Entornos Interactivos y en el Grado en Diseño en BAU, Centre Universitari d'Arts i Disseny de Barcelona. Universitat de Vic Universitat Central de Catalunya (UVicUCC). anna.carreras@bau.cat 